\title{
1 Fine scale structure of convective mixed layer in
}

2 ice-covered lakes

3 Sergey Volkov · Sergey Bogdanov · Roman

4 Zdorovennov - Galina Zdorovennova •

5 Arkady Terzhevik • Nicolay Palshin ·

6 Damien Bouffard • Georgy Kirillin

7 Received: date / Accepted: date

S. Volkov

Northern Water Problems Institute of the Karelian Research Centre of Russian Academy of Sciences (NWPI KarRC RAS)

Aleksander Nevsky st., 50, 185030 Petrozavodsk, Russian Federation

Tel.: +79535341611

E-mail: taranarmo@gmail.com

S. Bogdanov

Northern Water Problems Institute, the Karelian Research Center, Russian Academy of Sciences (NWPI KarRC RAS)

Petrozavodsk, Russian Federation

R. Zdorovennov

Northern Water Problems Institute, the Karelian Research Center, Russian Academy of Sciences (NWPI KarRC RAS)

Petrozavodsk, Russian Federation

G. Zdorovennova

Northern Water Problems Institute, the Karelian Research Center, Russian Academy of Sciences (NWPI KarRC RAS)

This document is the accepted manuscript version of the following article:

Volkov, S., Bogdanov, S., Zdorovennov, R., Zdorovennova, G., Terzhevik, A., Palshin, N., ... Kirillin, G. (2019). Fine scale structure of convective mixed layer in ice-covered lake. Environmental Fluid Mechanics, 19, 751-764. https://doi .org/10.1007/s10652-018-9652-2 
8 Abstract Nonstationary convection forced by distributed buoyancy sources is a

9 fundamental environmental fluid mechanics process, particularly in ice-covered

10 freshwater waterbodies. In this paper, we present novel field-based results that

11 characterise the diurnal evolution of the main energetics of radiatively-driven con-

12 vection in ice-covered lakes that is the radiatively-induced buoyancy flux, $\mathrm{B}$, and

13 the kinetic energy dissipation rate, $\varepsilon$. To estimate the spatiotemporal distribution

14 of $\varepsilon$, we applied scale similarity of the velocity structure functions to identify the

15 fine turbulence scales from high-frequency velocity measurements. The field study

16 was carried out at Lake Vendyurskoe, Russia, in April 2016. Small-scale velocity

17 fluctuations were measured using acoustic Doppler current profiler in a $2 \mathrm{~m}$ layer

18 beneath the ice cover. The method was proven to be valid for low-energy convec-

Petrozavodsk, Russian Federation

A. Terzhevik

Northern Water Problems Institute, the Karelian Research Center, Russian Academy of Sciences (NWPI KarRC RAS)

Petrozavodsk, Russian Federation

N. Palshin

Northern Water Problems Institute, the Karelian Research Center, Russian Academy of Sciences (NWPI KarRC RAS)

Petrozavodsk, Russian Federation

D. Bouffard

Surface Waters-Research and Management, Swiss Federal Institute of Aquatic Sciences and Technology (EAWAG)

Kastanienbaum, Switzerland

G. Kirillin

Leibniz-Institute of Freshwater Ecology and Inland Fisheries (IGB)

Berlin, Germany 
tion without mean shear. The inertial subrange, covering order of magnitude in the spatial domain, was identified by fitting the ${ }^{2} / 3$ scaling power law to the structure function method, thus confirming the regime of fully developed turbulence. The calculated rate of dissipation of turbulent kinetic energy $\varepsilon$ reaches values up to $3 \times 10^{-9} \mathrm{~m}^{2} \mathrm{~s}^{-3}$. Although a strong correlation between $\varepsilon$ and $\mathrm{B}$ was observed, $\varepsilon$ picks up about $1 \mathrm{~h}$ later after the onset of the heating-phase. This delay roughly corresponds to the turnover time of the energy containing eddies. We finally observed a decay of $\varepsilon$ at night, during the relaxation-phase, but, interestingly, the level remained above the statistical error.

Keywords convection · velocity structure function $\cdot$ inertial subrange $\cdot$ dissipation rate $\cdot$ acoustic Doppler profiling

\section{Introduction and problem statement}

Gravitationally driven convection is a crucial mechanism of energy and mass transport in geophysical flows. In particular, developed turbulent convection has been a challenging problem in the theory of turbulence for decades, and the idealized Rayleigh-Benard setup of the convection problem is often referred to as a classical model for turbulence development [18].

Originally the convection studies were motivated by atmospheric processes research [4, 5, 17, 20]. The key concept — the so-called "Deardorf convective scale" - was derived for the case, when convection is triggered by a boundary buoyancy flux. Another type of convection, particularly relevant in oceanological [1], 26, 34], and limnological [6] environments, is the convection driven by a volumetric source of gravitational instability. This energy supply particularly occurs during early 
42 spring in ice-covered freshwater lakes, where the bulk of water absorbs the solar

43 radiation penetrating the ice. Radiative heating triggers gravitational instabilities

44 in upper waters as long as the water temperature is below that corresponding

45 to the maximum density $\left(3.98^{\circ} \mathrm{C}\right)$. Convection then develops with virtually no

46 lateral boundary effects and with a fixed temperature at the upper boundary (ice-

47 water interface) that is isolated by ice from the wind action at the surface. In this

48 regard, convection due to solar heating in ice-covered lakes is a nearly ideal "natural

49 laboratory" for investigating the phenomenon without mean shear, relevant to the

50 geo- and astrophysical flows [6, 10, [25].

Recent autonomous in situ measurements of flow velocities and temperature

${ }_{52}$ have enabled direct observation of radiatively driven convection in ice-covered lakes [2, ए2, [3]. The derived data trigger the progress in studying the energetic

54 of the process, including estimations of buoyancy flux, dissipation rates, and mixing efficiency. In particular, Mironov et al. [21] adapted the well-known Deardorff convective velocity scaling (based on the surface buoyancy flux) to the case of 57 volumetrically distributed radiation source. Jonas et al. [9] performed an analy${ }_{58} \quad$ sis of the turbulent kinetic energy (TKE) budget using estimates of the energy

59 dissipation rate $\varepsilon$ obtained from temperature microstructure profiles.

The aim of this paper is to characterize the energetic properties of under-ice ${ }_{61}$ radiatively driven convection based on in situ velocity measurements. The obser62 vations were carried out in a small boreal lake during the early-spring warming ${ }_{63}$ period. The data obtained by an acoustic Doppler velocity profiler were used to de64 termine velocity structure functions and so estimate the spatiotemporal structure

65 of the rate of dissipation of kinetic energy $\varepsilon$. 
$\varepsilon$ is commonly used in in situ study to quantify the level of turbulence and the mixing efficiency [30]. Yet, the estimation of $\varepsilon$ remains very challenging in the field. For example, the so-called temperature microstructure method (TMM) is based on the fitting of the high frequency wavenumber spectrum of temperature with theoretical Batchelor spectrum [I, 9, 20]. The inertial dissipation method

71 (IDM) makes it possible to estimate dissipation rates from measured time series 72 of current velocities, applying Taylors frozen flow hypothesis [20]. Moreover, the 73 impossibility to distinguish the streamwise to the spanwise velocity fluctuations 74 in the beam velocity coordinate adds an uncertainty of $(4 / 3)^{3 / 2} \approx 0.65$ when 75 using IDM [20]. In our study we estimated $\varepsilon$ by the direct analysis of the inertial 76 interval. The variability of $\varepsilon$ with depth and its dependence on convective forcing 77 by solar radiation on diurnal scales were quantified.

The paper is organized as follows. The framework of the scaling analysis for ve-

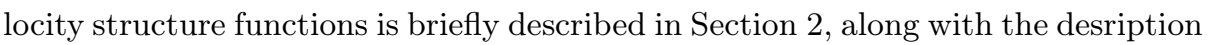

80 of the study site, instrumental setup, and the design of the experiment. Section $\$$ is

${ }_{81}$ devoted to the observed bulk characteristics of convection. In Section $\square$ we present

82 the main results, including estimations of $\varepsilon$. The discussion is provided in Section 15 .

83 The main outcomes and findings are summarized in the Section $\mathbf{6}$.

\section{Major scales, field experiment setup, and data analysis methods} Scaling of turbulent velocity fluctuations. Kolmogorov [14] developed theory of

86 isotropic turbulence based on the concept of direct energy cascade. One of the corollaries of this theory is the ${ }^{2} / 3$ law' that characterizes the two-point structure

${ }_{88}$ functions $D_{i j}(\vec{x}, \vec{r}, t)=\left\langle\left(u_{i}(x+r, t)-u_{i}(x, t)\right)\left(u_{j}(x+r, t)-u_{j}(x, t)\right)\right\rangle$ of velocity 
89 components increments at a distance $r$. In the inertial subrange

$$
D_{L L}=C \varepsilon^{2 / 3} r^{2 / 3} ; r_{d} \ll r \ll r_{0}
$$

90

91

\begin{tabular}{|c|c|c|c|c|}
\hline Value $\backslash$ subrange & dissipative & inertial & energy containing & external \\
\hline size & $r_{d}$ & $r_{i}$ (upper boundary) & $r_{0}$ & $h-\delta$ \\
& & $\lambda$ (Taylor microscale) & & \\
\hline time & $\tau_{d}$ & & $\tau_{0}$ & $\tau^{*}$ \\
\hline velocity & $v_{d}$ & & $v_{0}$ & $w^{*}$ \\
\hline
\end{tabular}

Table 1 The list of variables

Here, $D_{L L}$ is the longitudinal (for the velocity component which is collinear with $\vec{r}$ ) structure function, $\varepsilon$ is the TKE dissipation rate per unit mass, $C \approx 2.09$ [ 22 ,

[27] is the Kolmogorov constant, $r_{d}$ is the dissipative length scale, and $r_{0}$ is the energy-containing scale. The transverse structure function $D_{N N}$ (for the velocity components which are perpendicular to $\vec{r}$ ) obeys the same relation with transformed constant $C^{\prime}={ }^{4} /{ }_{3} C$.

The calculation of SF requires a proper experimental setup as spatial and registration intervals of measurements play crucial role. The list of variables involved in the procedure is presented in Table $\mathbb{m}$.

The list of varial

In particular, it is only possible to extract the inertial subrange lower boundary from data with spatial resolution close to $r_{d}$. Moreover, the ensemble averaging in (四) should remain close to the energy containing eddies turnover time $\tau_{0}$. Here both the value of $\tau_{0}$ and temporal discreteness of measurements are important to achieve the statistical reliability.

Adopting the range of the energy dissipation rates $\varepsilon=10^{-10}-10^{-8} \mathrm{~m}^{2} \mathrm{~s}^{-3}$ reported previously for ice-covered lakes $[9,[2]$ and the water kinematic viscosity 
$\nu \approx 1.6 \times 10^{-6} \mathrm{~m}^{2} \mathrm{~s}^{-1}$, the dissipative scales for length $r_{d}$, time $\tau_{d}$, and velocity $v_{d}$, characterizing the lower boundary of the inertial interval of the turbulent energy

cascade can be estimated as

$$
\begin{gathered}
r_{d}=\left(\nu^{3} / \varepsilon\right)^{1 / 4} \approx(5-10) \cdot 10^{-3} \mathrm{~m} ; \\
v_{d}=(\nu \varepsilon)^{1 / 4} \approx(1-3) \cdot 10^{-4} \mathrm{~m} \mathrm{~s}^{-1} \\
\tau_{d}=(\nu / \varepsilon)^{1 / 2} \approx(10-100) \mathrm{s}
\end{gathered}
$$

The turnover time $\tau_{0}$ (and, correspondently, the time averaging interval) one can roughly estimate as the ratio of convectively mixed layer (CML) thickness $h-\delta$ and convective velocity scale $w^{*}$, which is governed by the negative buoyancy flux [4, 21]:

$$
w^{*}=\left(-[h-\delta] B_{R}\right)^{1 / 3}
$$

Here $h$ and $\delta$ - lower and upper boundaries of CML,

$$
B_{R}=B(\delta)+B(h)-2(h-\delta)^{-1} \int_{\delta}^{h} B(z) \mathrm{dz}
$$

$B(z, t)=\beta(z, t) I(z, t)\left[\mathrm{m}^{2} \mathrm{c}^{-3}\right]$ is the vertically distributed source of buoyancy produced by absorption of solar radiation flux $I(z)\left[\mathrm{K} \mathrm{m} \mathrm{s}^{-1}\right]$, and $\beta$ is the buoyancy parameter $\left[\mathrm{m} /\left(\mathrm{Ks}^{2}\right)\right]$. For fresh water with temperature $\theta$ close to $\theta_{r} \approx 277 \mathrm{~K}$ the last parameter is approximated $\beta=\alpha_{0} g\left(\theta-\theta_{r}\right)$, where $\alpha_{0} \approx 1.65 \times 10^{-5} \mathrm{~K}^{-2}$.

The estimation of the inertial interval upper boundary $r_{i}$ is a more subtle issue. This boundary lies within the interim range of scales between inertial and energycontaining intervals. Its direct estimations from experimental data are questionable, taking into account the accuracy of measurements currently available (see Figures 4 and 5 below). However the simple estimations of the so-called Taylor microscale $\lambda=\sqrt{15 \frac{v}{\varepsilon}} w^{*}$ are easily available for evaluation the inertial interval boundary [24]. For typical values (a few $\mathrm{mm} / \mathrm{s}$ ) of convective velocity $w^{*}$ the lengthscale takes the values around a few tens of centimeters. 


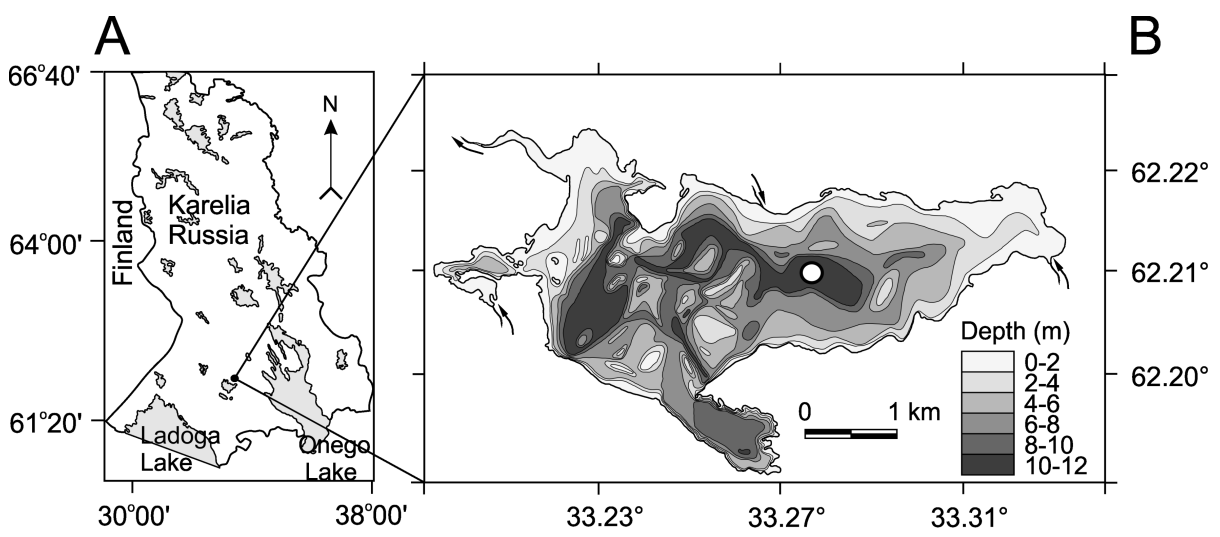

C
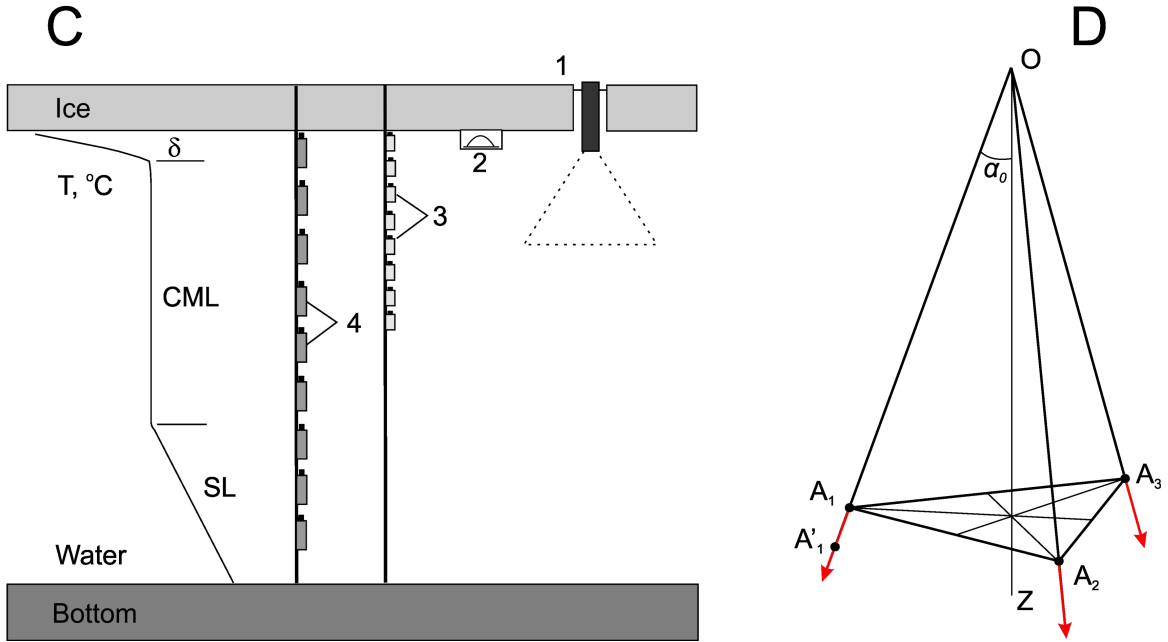

Fig. 1 (A) geographic location and (B) bathymetry map of Lake Vendyurskoe with the location of the measurement site (white circle); (C) instrumental setup and a sketch of the thermal structure during under-ice convection (see main text for abbreviations), 1 - Aquadopp, 2 - Under-ice pyranometer, 3 - PAR-chain, 4 - TR-chain; (D) Beams geometry: the angle $\alpha_{0}=25^{\circ}$ specifies the beams' orientation off the vertical and the azimuth orientation of three beams is symmetric.

Study site and field experiment setup. The field experiment was carried out in the central deep-water part of Lake Vendyurskoe, Russia $\left(62^{\circ} 20^{\prime} N, 33^{\circ} 20^{\prime} E\right)$ (Fig. Ш, B), on 8-13 April 2016. Lake Vendyurskoe is a shallow polymictic lake of glacial origin in northwestern Russia. The lake area is $10.4 \mathrm{~km}^{2}$, the average and maximal depths 

$153 \pm 4 \% F S$, resolution $\left.1 \mu \mathrm{mol} \mathrm{m}^{-2} \mathrm{~s}^{-1}\right)$. The eight PAR sensors were attached to the are $5.3 \mathrm{~m}$ and $13.4 \mathrm{~m}$, respectively; the maximal length is $7 \mathrm{~km}$; and the average width is $1.5 \mathrm{~km}$. The water transparency (depth of Secchi disk) is $3-4 \mathrm{~m}$. Ice-on generally occurs by late November-early December, ice-off occurs by early-middle May; and the maximal snow-ice sheet thickness is $0.6-0.8 \mathrm{~m}$ [32].

Measurements of water temperature and solar radiation flux at the lower boundary of ice and within a water column, as well as direct measurements of the currents, were obtained during the period from 8 to 13 April 2016. The schematic

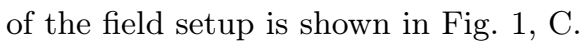

A $2 \mathrm{MHz}$ HR Aquadopp current velocity profiler (Nortek AS, Norway) was frozen into the ice with the acoustic transducer head looking downward into the water column ( 1 on the Fig. 四, C). The Aquadopp settings were as follows: $2.1 \mathrm{~m}$ pulse distance, $1.9 \mathrm{~m}$ profile range, 38 cells, $60 \mathrm{~s}$ burst interval, 16 samples per burst, $4 \mathrm{~Hz}$ sampling rate, and $5 \mathrm{~cm}$ cell size.

Measurements of the three components of current velocity in the $2 \mathrm{~m}$ layer immediately under the ice were accompanied by observations on the under-ice solar radiation and water temperature.

Downwelling planar irradiance at the ice base was measured with an M-80m universal pyranometer produced in Russia (accuracy $1 \mathrm{~W} \mathrm{~m}^{-2}$, registration interval $1 \mathrm{~min})$. This device was mounted onto a piece of foam with positive buoyancy and placed under the ice at a distance of $1.5-2 \mathrm{~m}$ from the ice hole (2 on the Fig. $\mathrm{W}, \mathrm{C})$.

We also measured the PAR (photosynthetically active radiation) flux at the lower boundary of the ice and within a water column using sensors JFE Alec MkV-

L (Alec Electronics, Japan, 390-690 nm, range 0-2000 $\mathrm{mmol} \mathrm{m}^{-2} \mathrm{~s}^{-1}$, accuracy 
fishing line at intervals of $0.5-1 \mathrm{~m}$ to a depth of $5 \mathrm{~m}$, with the top sensor located directly under the ice (3 on the Fig. $\mathrm{\square}, \mathrm{C})$.

Assuming local exponential decay of PAR in the water column as a function of depth by $E_{d}(z)=E_{d}\left(0^{-}\right) e^{\left(-K_{w} z\right)}$, where $E_{d}\left(0^{-}\right)$and $E_{d}(z)$ are downwelling solar irradiance at the water-ice interface and at the depth $z$, respectively, we calculated the attenuation coefficient $K_{w}$ between two depth $z_{1}$ and $z_{2}$ from the measured values of PAR at different depths within a water column: $K_{w}\left(z_{2}, z_{1}\right)=$ $\frac{-1}{z_{1}-z_{2}} \ln \frac{E_{d}\left(z_{1}\right)}{E_{d}\left(z_{2}\right)}$

The thermistor chain (4 on the Fig. 四, C) was fixed to the ice and anchored. Temperature sensors (43 TR sensors, RBR Ltd, accuracy $\pm 0.002^{\circ} \mathrm{C}$, registration interval 10 seconds) were fixed at an interval of $0.25 \mathrm{~m}$ from the lower boundary of the ice to the bottom. These temperature sensors allowed the boundaries of the convective layer to be determined and traced their dynamics during the measurement period. A schematic representation of the temperature profile with advanced convection is shown in Fig. $\mathrm{W}, \mathrm{C}$, where $\delta$ is the the under-ice gradient layer, CML is the convectively mixed layer, and SL is the stratified layer.

Velocity fluctuations and velocity structure function. Two-point velocity structure functions $D_{i j}$ were chosen as the key characteristics for the analysis of the finescale convection. Measurements of the velocity components were performed using the Aquadopp HR-Profiler (Nortek, Norway). The appropriateness of acoustic Doppler profilers for the derivation and analysis of $D_{i j}$ was previously demonstrated in atmospheric and marine studies [3I]. However, a correct choice of the spatial and registration interval is crucial for subsequent estimations and depends on the characteristic scales of specific turbulent process. 
The measurement configuration allows us to derive only the longitudinal structure functions $D_{L L}$, because, for each beam, only the along beam component $b_{i}$ of the current velocity is estimated from the Doppler shift. Hence, for each depth $z$, only the functions

$$
D_{L L}\left(z, r_{i}\right)=\left\langle\left(b_{i}^{\prime}\left(A_{i}^{\prime}\right)-b_{i}^{\prime}\left(A_{i}\right)\right)^{2}\right\rangle
$$

were available for the statistical analysis of velocity increments. Here, $r_{i}=A_{i} A_{i}^{\prime}$ is the spacing between points along a single acoustic beam (Fig. W, D), $z$ is the vertical coordinate of the reference point $A_{i}$ counted out from the lower surface of the ice, the brackets \langle\rangle indicate an ensemble average, and $b_{i}^{\prime}$ is the velocity with the temporal mean removed.

The choice of spatial resolution is one of the key issues when implementing Eq. (피). Equation (可) is valid, when the spacing between measurement points $r$ should fall into the inertial interval of turbulence $r_{d} \ll r \ll r_{0}$. Consequently, the vertical spacing of the velocity measurements was set to $5 \mathrm{~cm}$. This value is almost an order of magnitude higher than the expected Kolmogorov length scale $r_{d}$, thus we assume the turbulence to be developed enough for the inertial interval to extend over this scale. In the absence of strong mean currents, acoustic velocity measurements were performed using pulse-to-pulse coherent processing [16], which allows a significant reduction of acoustic noise at the cost of the profiling range. The $2 \mathrm{MHz}$ HR Aquadopp profiler in the pulse-to-pulse coherent mode scans a $2 \mathrm{~m}$ thick layer with a blind zone in the first $15 \mathrm{~cm}$ from the device head. Hence, the variable $r_{i}$ in Eq. (四) contained 38 points. The along-beam coordinate varied in the range $(0.17,2.21) \mathrm{m}$. 
221 (Fig. 四, C)

$$
D_{L L}=N+C \varepsilon^{2 / 3} r^{2 / 3}
$$

which includes the noise estimation as $N=2 \sigma_{N}^{2}$; the variance $\sigma_{N}^{2}$ depends on instrumental noise of the ADCP and non turbulent velocity fluctuations. In general, $\sigma_{N}^{2}$ varies with depth and the distance $r$, so the problem of noise exclusion is not straightforward. To ensure statistical reliability, two-step time averaging was implemented, using the "burst" measurement mode of the instrument. Thus, profiles were recorded at time intervals of $60 \mathrm{~s}$. Considering the device capacity limits, we restricted the number of samples within each burst to $n_{p}=16$. That is, with a ping frequency of $4 \mathrm{~Hz}$, the active period was $4 \mathrm{~s}$. For each burst, preliminary averaging of the velocity records (over the number of pings within the burst) was carried out to account for Gaussian statistics and to remove the local noise. The resulting "refined instantaneous velocities" were used for calculation of structure function by ensemble "inter-burst" averaging.

\section{Observed bulk characteristics of convection and their relationship to}

\section{the mixing scales}

Vertical thermal structure and subsurface solar radiation. The vertical structure of the water column under the ice is characterized by three layers during the penetrative convection phase: a thin surface under-ice gradient layer of thickness $\delta$, a convectively-mixed layer CML, and a stably stratified quiescent layer SL [6, 21] 


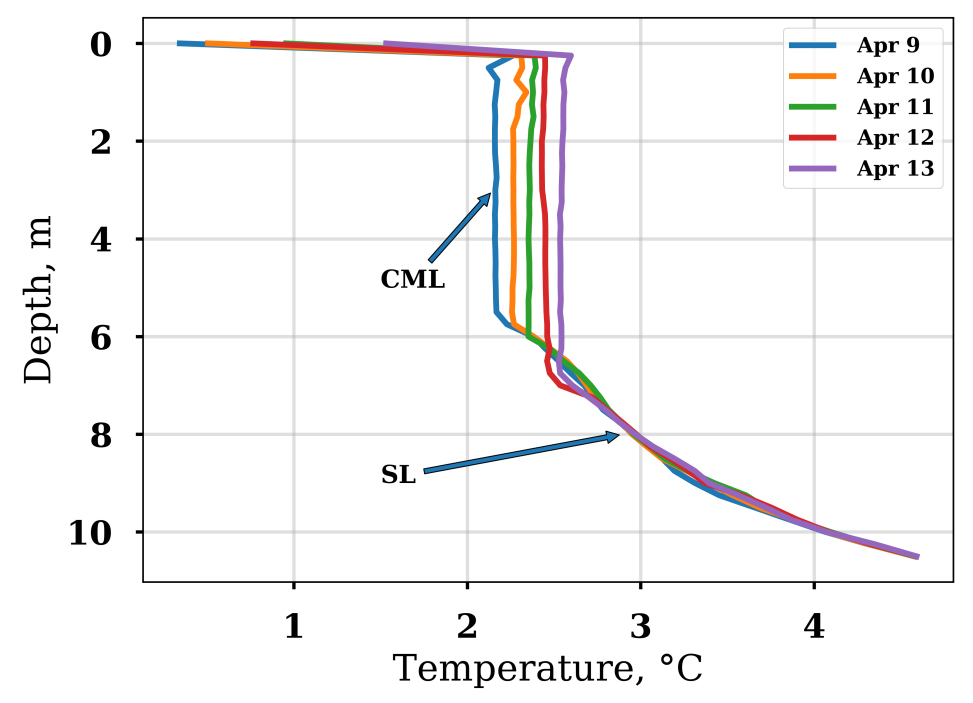

Fig. 2 The vertical temperature profile dynamics during April 09-12, 2016

The upper boundary of the CML was observed at depths of $0.5-0.6 \mathrm{~m}$. The under-ice gradient layer on top of the CML was clearly recognizable during the measurements, with temperature gradients reaching $10^{\circ} \mathrm{C} \mathrm{m}^{-1}$. The temperature of CML increased from $2.11^{\circ} \mathrm{C}$ to $2.53{ }^{\circ} \mathrm{C}$, and its lower boundary deepened from $5.5 \mathrm{~m}$ to $6.75 \mathrm{~m}$ during the measurement period (Fig. $\nabla)$.

The daytime maxima of downwelling planar irradiance at the water-ice interface exceeded $150 \mathrm{~W} \mathrm{~m}^{-2}$ on $9-11$ and 13 April 2016, and they were only $100 \mathrm{~W} \mathrm{~m}^{-2}$ on 12 April 2016 (Fig. B, A). The PAR flux decreased rapidly with increasing depth and did not exceed $20 \mathrm{Wm}^{-2}$ at a depth $>2 \mathrm{~m}$ (Fig. B, B). The maximal values of $K_{w}$ were confined to the under-ice layer $0-0.5 \mathrm{~m}$ and varied between $1.3-1.9 \mathrm{~m}^{-1}$. The values of $K_{w}$ were $0.8-1.2 \mathrm{~m}^{-1}$ at a depth of $1-4 \mathrm{~m}$. The temperature increse and deepening of the lower boundary of the convective layer occurred in the daytime against the background of solar heating (Fig. B, C). 

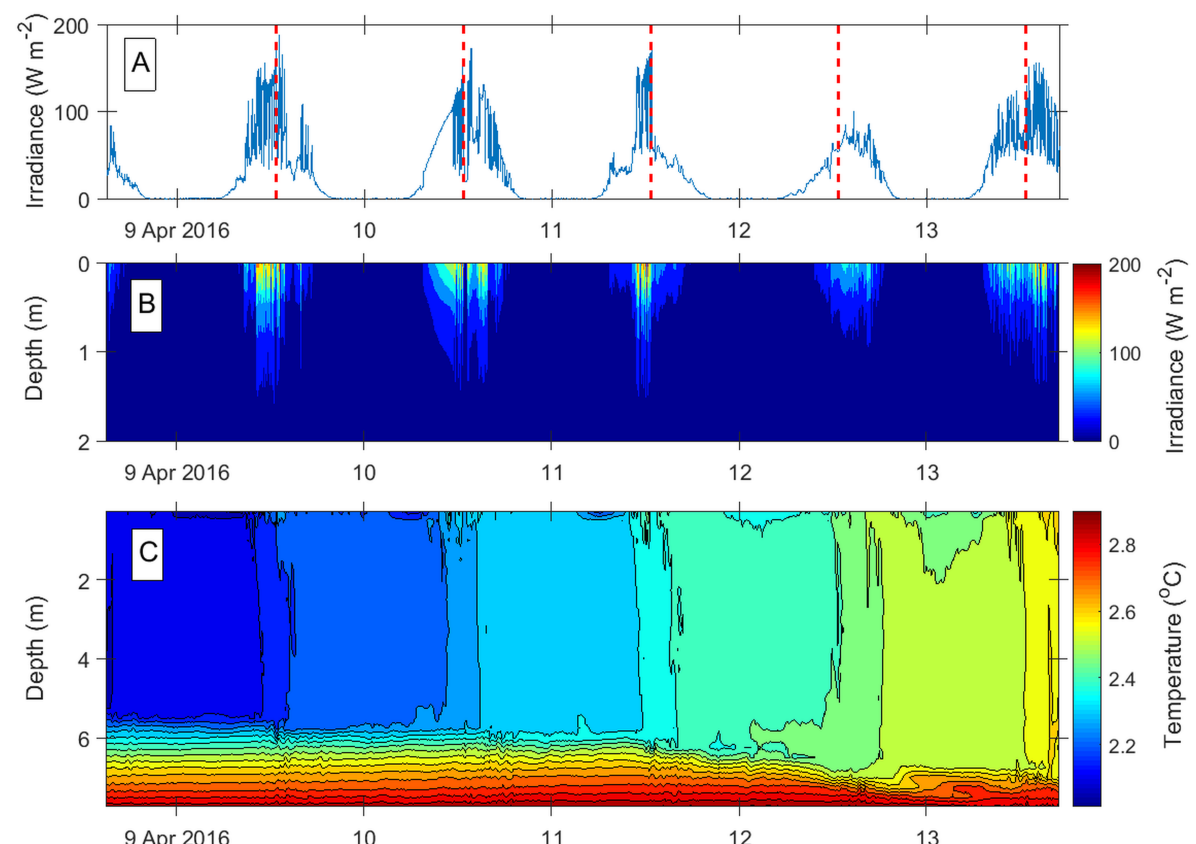

Fig. 3 Under-ice downwelling planar irradiance (A) at the lower boundary of ice and (B) at different depths into a water column during 8-13 April 2016; water temperature (C) at the same period (the figure shows a depth range $0.25-7.75 \mathrm{~m}$ ). The red dotted lines in panel A represent the time of astronomical noon (12:40).

Physical scales of convection. The vertical scale $r_{0}$ of energy-containing convective eddies is restricted by the CML thickness, $h-\delta \approx 5 \mathrm{~m}$. From Eq. (更), we obtained the typical value $v_{0} \sim(1-5) \mathrm{mms}^{-1}$, which in turn provides an upper limit estimate for the turnover time $\tau_{0} \approx 5 \times 10^{3} \mathrm{~s}$, i.e., $1.5 \mathrm{~h}$. This value is an order of magnitude smaller than the diurnal period, but close to that of typical daytime variations of the radiation flux. Hence, this thick CML (with a depth exceeding $1 \mathrm{~m})$ cannot be viewed as stationary, even during daytime.

The well-developed character of convective mixing under ice is supported by the observed high degree of homogeneity within the CML (Fig. [ॄ). Further quantitative 
support is provided by the estimation of the Rayleigh number. Convection driven by the volumetric buoyancy flux is characterized by the so-called modified Rayleigh number $R[\overline{[},[28]$ :

$$
R=\frac{\beta g(h-\delta)^{5} H}{k^{2} v}
$$

which is a flux-based counterpart of the classical Rayleigh-Bénard number.

Here $k$ is the molecular thermal diffusivity. The characteristic temperature scale $\theta^{*}=H(h-\delta)^{2} \mathrm{k}^{-1}$ related to the internal heating rate $H=\partial I / \partial z\left(\mathrm{~K} \mathrm{~s}^{-1}\right)$ is used instead of the initial temperature difference $\Delta \theta$ between the top and bottom boundaries. Using the typical observed subsurface radiation values $30 \mathrm{~W} / \mathrm{m}^{2}$ for the radiation heat flux $Q$ and a light extinction coefficient on the order $1 \mathrm{~m}^{-1}$ [3:3], we estimated the heating rate to be $H \approx 3 \times 10^{-6} \mathrm{~K} \mathrm{~s}^{-1}$. Substituting this typical value of $H$ in Eq. (3), we obtained the estimate $R \sim 10^{14}$. This value exceeds by several orders of magnitude the value $10^{10}$ previously reported as corresponding to the fully developed convective regime in DNS studies [3, [] of internally heated convection.

\section{Structure functions. Inertial interval. Estimations of $\varepsilon$}

Computations of the structure function $D_{L L}$ were carried out for each of the three beams. In some cases, the results for different beams appeared to be significantly different, although all of the curves demonstrated similar shapes. We assumed that the flow was homogeneous in the horizontal plane and local isotropy in fine-scale structures and thereby averaged our results over the three beams. Under these assumptions, the scattering of experimental data was sufficiently reduced and the 

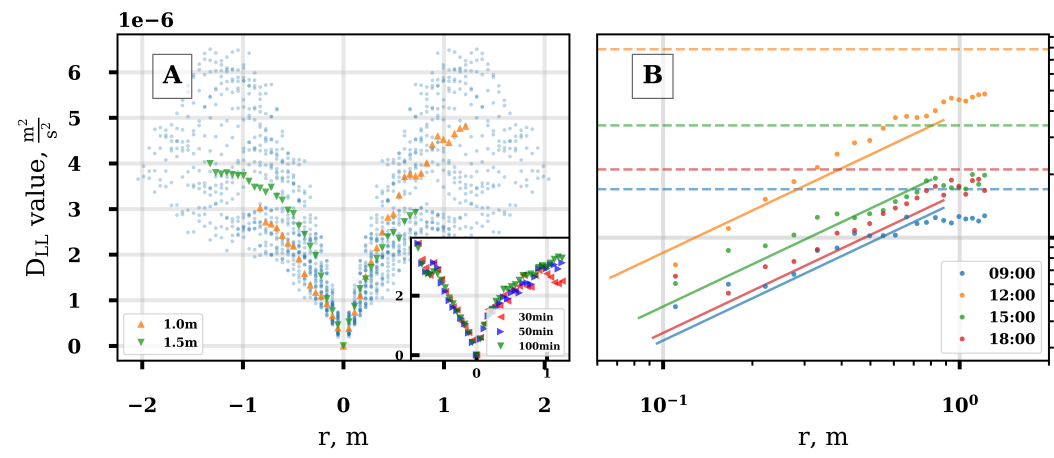

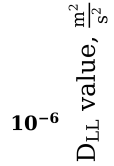

Fig. 4 A: Three-beams averaged structure functions $D_{L L}$ for the full series of reference points; 11 April 2016, 12:00, 100 min averaging; the orange and green triangles represent the structure functions for reference points 1.0 and $1.5 \mathrm{~m}$ correspondently; inset: structure functions sequence for different periods of averaging, 9 April 2016, 12:00, $1.5 \mathrm{~m}$ depth; B: $D_{L L}$ evolution during daytime of 11 April 2016; the reference point (the axis origin) is at a depth of $1 \mathrm{~m}$ beneath the ice cover.Thick segments show the extension of the inertial interval for each line, derived as quasilinear parts of the corresponding curves

tendency of the structure functions $D_{L L}$ to follow a self-similar shape became more evident (Fig. 因 A).

To obtain reliable quantitative results, the computations of the structure functions were carried out for different values of $\tau$, starting with $0.2 \tau_{0}$ and successively increasing up to $\tau_{0}$. We observed a "saturation" at a threshold $\tau \approx 0.5 \tau_{0}$ with the shape of $D_{L L}$ approaching an asymptotic form (Fig. 四 A, inset).

To check the presence of the Kolmogorov inertial range and, accordingly, the validity of the "2/3 law" in the observed regime of free convection driven by volumetric buoyancy flux, we investigated the dependence of $D_{L L}(r)$ on the parameter $r$ in the following way. The inertial range was identified as the linear part of the function $D_{L L}\left(r^{2 / 3}\right)$ using an iterative procedure. First, we approximated the full set of experimental points by linear dependence and used the least squares 
277 method to calculate the slope and consequently $\varepsilon$, as well as the root-mean-square error $\Delta \varepsilon$. In the next step, the point with maximal $r$ was removed and the procedure repeated. The iteration cycle ceased on a step $i$ for which the condition $\left|\varepsilon_{i}-\varepsilon_{i-1}\right|<\Delta \varepsilon_{i}$ was fulfilled. Fig. 田 B illustrates the extent and daytime variations of the inertial interval. The dashed lines represent the asymptotic values $2\left\langle b^{2}\right\rangle$ of the structure functions (see Eq. (3)).

Estimations of the energy dissipation rate $\varepsilon$.

\begin{tabular}{|l|l|l|}
\hline Time & $(\varepsilon \pm \Delta \varepsilon) 10^{-9} m^{2} s^{-3}$ & $r_{i} / r_{d}$ \\
\hline Apr 09 09:00 & $1.71 \pm 0.15$ & 126 \\
Apr 09 12:00 & $1.66 \pm 0.12$ & 125 \\
Apr 09 15:00 & $2.10 \pm 0.33$ & 133 \\
Apr 09 18:00 & $0.69 \pm 0.07$ & 101 \\
Apr 10 09:00 & $1.80 \pm 0.19$ & 128 \\
Apr 10 12:00 & $1.28 \pm 0.16$ & 117 \\
Apr 10 15:00 & $2.10 \pm 0.14$ & 133 \\
Apr 10 18:00 & $1.13 \pm 0.12$ & 114 \\
Apr 11 09:00 & $0.72 \pm 0.08$ & 102 \\
Apr 11 12:00 & $3.18 \pm 0.57$ & 147 \\
Apr 11 15:00 & $1.09 \pm 0.06$ & 113 \\
Apr 11 18:00 & $0.85 \pm 0.20$ & 106 \\
Apr 12 09:00 & $0.44 \pm 0.04$ & 90 \\
Apr 12 12:00 & $0.84 \pm 0.05$ & 105 \\
Apr 12 15:00 & $2.11 \pm 0.35$ & 133 \\
Apr 12 18:00 & $0.64 \pm 0.06$ & 99 \\
\hline
\end{tabular}

Table 2 Energy dissipation rates and inertial interval extent calculated for the daylight hours, 9-12 April 2016. Depth $1 \mathrm{~m}$. 


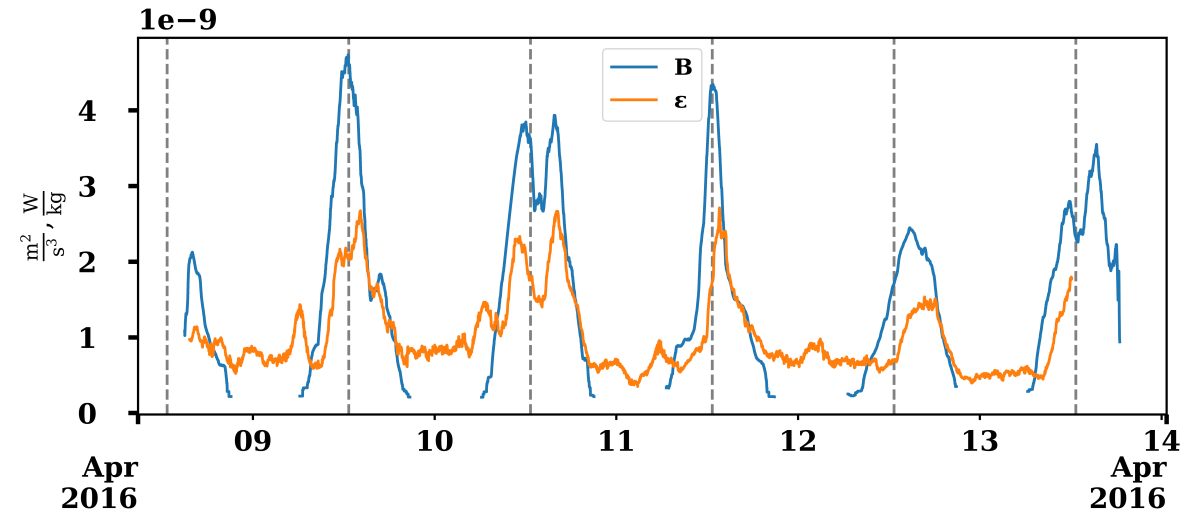

Fig. 5 Buoyancy production term ( $B$, blue line) and kinetic energy dissipation rate $(\varepsilon$, orange line) dynamics; 9-13 April 2016, dashed lines are noon times (12:40).

The values of $\varepsilon$ varied around $10^{-9} \mathrm{~m}^{2} \mathrm{~s}^{-3}$, in qualitative agreement with previous estimates $[9,[2]]$. The mean, median, and maximal values of depth-averaged $\varepsilon$ (in units $10^{-9} \mathrm{~m}^{2} \mathrm{~s}^{-3}$ ) were calculated as $1.15,1.07$, and 3.27 for daytime and $0.41,0.37$, and 1.55 at night.

The computational results for a depth of $1 \mathrm{~m}$ covering 4 full days of observations, 9-12 April 2016, are presented in Table [. The inertial interval was clearly identified throughout most part of the daylight period. For each time its extension was calculated during iteration procedure as described above; its upper boundary $r_{i}$ had a typical absolute value of $\sim 0.5 \mathrm{~m}$ (see the last column of Table $\square$ ).

Therefore, the inertial subrange appears to be wide enough to contain the measurement discretization, supporting the initial choice of the measurement intervals based on the general scales of convective energy production and dissipation.

Apr
orcing, as demonstrated by both the extension of inertial subrange and $D_{L L}$ val- 

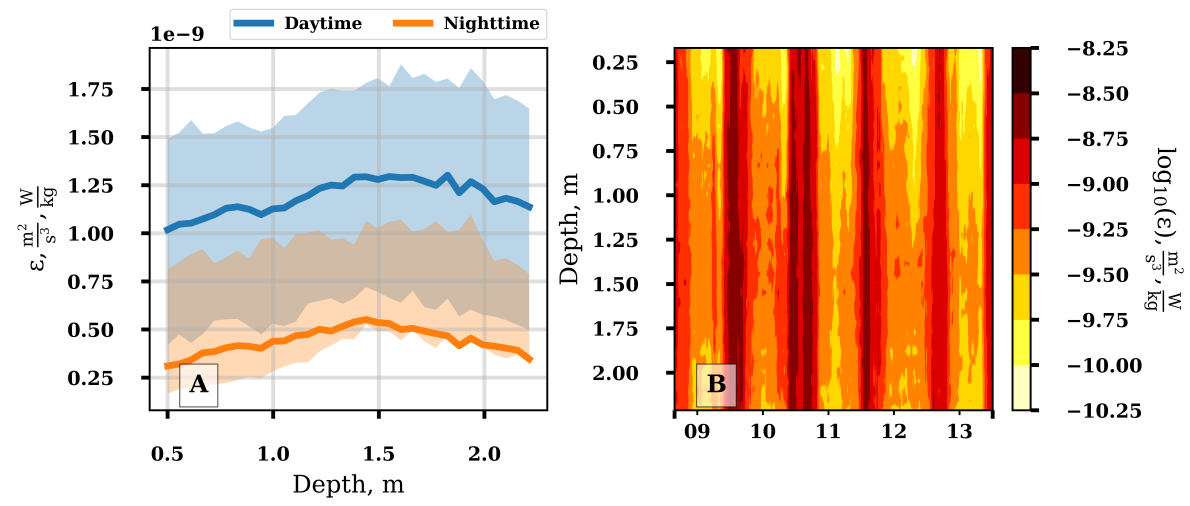

Fig. 6 Time and depth variations of the kinetic energy dissipation rate: the left panel presents the dependence of $\varepsilon$ averaged over the entire period of observations (on depth); the shaded areas are restricted by the curves of quantile functions corresponding to the probabilities 0.25 and 0.75. Right panel presents evolution $\varepsilon$ during 9-13 April, 2016.

ues (Fig. 田 B). Quantitatively, the mean dissipation rates within the convective layer agreed well with the buoyancy production term $B_{R}$ calculated by (Eq. \). Both production and dissipation followed the same diurnal patterns (Fig. 15). Neither a nighttime threshold (complete ceasing of $\varepsilon$ at low radiation fluxes) nor the cumulative effects of radiation on the over-diurnal time scales on $\varepsilon$ were observed. However, a clearly pronounced time delay persisted between $B_{R}$ and $\varepsilon$ (Fig. [5). The value of this delay was calculated by studying the dependence of covariance between $B_{R}$ and $\varepsilon$ for different time shifts. The shifts, corresponding to the maximal value of the covariance, varied from 68 to 135 minutes for different days, with the mean value close to 77 minutes.

Noteworthy, the nighttime values of $\varepsilon$, while sufficiently reduced compared with the daytime values, remained above the statistical error despite zero buoyancy

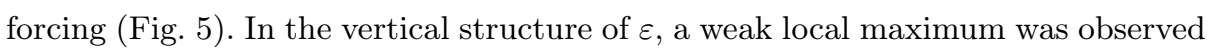
at a depth close to $1.5 \mathrm{~m}$, followed by a slight monotonic decrease with depth 
(Fig. 6). No clear tendency in dissipation rates at the ice base was recognized because of high measurement errors near the solid boundary.

\section{Discussion}

Our observations demonstrate that, despite the relatively slow convective motions, the Kolmogorov scaling for the velocity structure functions holds for convection without mean shear, as observed in ice-covered lakes. Such a fully developed convective regime suggests a strong cascade of turbulent eddies from larger to smaller scales. With typical values of the TKE dissipation rates of $O\left(10^{-9}\right) \mathrm{W} \mathrm{kg}^{-1}$, the extent of the observed inertial subrange spanned over a decade of scales during daytime, with the upper boundary around few tens of centimeters. To confirm the validity of the Kolmogorov paradigm in these conditions, the additional issue needs to be checked beyond the $2 / 3$ scaling exponent: the local isotropy. One way of verifying the fine-scale isotropy is to compare the longitudinal and transverse structure functions; the relationship $D_{N N} / D_{L L}=4 / 3$ should be valid for the isotropic case $[22]$. Another method of inspection is based on a comparison of correlations along different spacing directions for the same velocity components. Both comparisons are not directly accessible with the present technique, as only the "beam" components of velocity are available from the acoustic profilers.

Observations of the velocity structure functions at a high registration interval provide a way to determine the characteristic temporal and spatial scales of the inertial turbulent cascade as well as their variations due to the forcing nonstationarity. Our results suggest that the upper boundary $r_{i}$ of the inertial interval at the spatial domain scale remains nearly constant against the dissipative Kolmogorov 
length scale as $r_{i}=A \cdot r_{d}$, where $A=(130 \pm 20)$. This upper boundary of the inertial range also corresponds to the lower boundary of the energy-containing interval. Hence, taking the derived ratio $r_{i} / r_{d}$ into account, we can now estimate the lower bounds of the typical velocity $v_{0}$ of large eddies and their "turnover time" $\tau_{0}$. Indeed, within the inertial interval, the energy flux $v_{0}^{3} / r_{0}$ remains constant and its value is equal to $\varepsilon$, so $v_{0} \sim\left(\varepsilon \cdot r_{0}\right)^{1 / 3}=A^{1 / 3} \cdot v_{d}$. Then, the estimation of $\tau_{0}$ is:

$$
\tau_{0}=r_{0} / v_{0}>A^{2 / 3} \cdot \tau_{d} \sim 20 \mathrm{~min}
$$

The "external" time macroscale of the convective motions can in turn be estimated as $\tau^{*}=h \cdot w^{*-1}$. The typical values of $h \approx 6 \mathrm{~m}$ and $B_{r} \approx 10^{-9} \mathrm{~m}^{2} \mathrm{~s}^{-3}$ yield $w^{*} \approx 1.8 \mathrm{~mm} \mathrm{~s}^{-1}$ and $\tau^{*} \approx 1 \mathrm{~h}$.

This value agrees with the time delay of $\sim 1 \mathrm{~h}$ between the production of the negative buoyancy by the solar radiation and the dissipation of the TKE. By this, $\tau^{*}$ can be interpreted as the "adjustment" time, so that for a suddenly applied constant buoyancy forcing, the convective energy budget becomes nearly steady-state after a period exceeding $\tau^{*}$. The apparent proximity of the convective micro- and macroscales $\tau_{0}$ and $\tau^{*}$ suggests that the macroscale $h w^{*-1}$ describes rather well the nonstationarity of the convection at variable buoyancy forcing $B_{R}$. Similar conclusions were derived by Nieuwstadt and Brost [2:3] using Large Eddy Simualtions to show that $h w^{*-1}$ is the governing time scale for the decay of the kinetic energy after the buoyancy forcing has ceased. The detected upper boundary of the self-similarity interval in the velocity structure domain $r_{0} \approx 130 r_{d}$ corresponds to $h r_{0}^{-1} \approx 6$, where $r_{0}^{-1}$ can be interpreted as the wavenumber of the energy pumping into turbulent motions. This value is slightly less than $h r_{0}^{-1} \approx 10$ reported in convection driven by the boundary heat flux [II]. One can suggest that con- 
vection with the volumetric buoyancy source allows generation of larger turbulent eddies. However, this suggestion requires further justification by observations of small-scale structure and/or by eddy-resolving numerical simulations.

The vertical distribution of the TKE dissipation rate is commonly assumed to be quasi-homogeneous within convective boundary layers []. and this is also supported by earlier LES modeling experiments and observations in ice-covered lakes using temperature microstructure [9, 21]. However, we observed a slight increase in the dissipation rate toward the ice base in the bulk of the $2 \mathrm{~m}$ thick layer covered by measurements. Similar $\varepsilon$ profiles were reported in the convective atmospheric planetary boundary layer [15.5]. Moreover, the present observations provide insight into the mixing conditions close to the ice-water interface at the boundary between the CML and the thin stably stratified layer above; these have been unavailable in previous studies. Here, a distinct upward increase of $\varepsilon$ was observed in the upper $0.5 \mathrm{~m}$ of the water column. Again, such strengthening of energy dissipation close to the boundary was observed by Lenschow et al. [15] in atmospheric convection due to surface buoyancy flux; they found that, at distances $z<0.2 \mathrm{~h}$ from the boundary, the TKE dissipation rate behaves as $\varepsilon \propto z^{-1}$, akin to the "wall turbulence" driven by the mean velocity shear. Our results qualitatively agree with the atmospheric data. In the particular case of under-ice convection, the intensification of turbulent mixing near the ice-water interface should play an important role in heat transport from the water column to the ice cover, accelerating ice melting. Also, in contrast to the atmospheric case, the energy transport at the top of the CML is affected - apart from the solid boundary — by the strong stratification in the stratified interfacial layer under ice, which can include generation of internal waves by convective motions. The complicated interplay of boundary shear, 
waves, and convective turbulence deserves a deeper future investigation as it has potentially important value for understanding the physics behind spring melting of seasonal ice.

\section{Conclusions}

Our study of the fine-scale velocity structure in CMLs demonstrates the applicability of acoustic Doppler profiler to estimate (i) the rate of dissipation of turbulent kinetic energy in a radiatively-driven convectively mixed layer and (ii) the range of the inertial subrange in the spatial domain. The results revealed the presence of the inertial interval with a scaling exponent close to $2 / 3$. During daytime, the energy dissipation rate remains around $3 \times 10^{-9} \mathrm{~W} \mathrm{~kg}^{-1}$. The inertial interval upper boundary $(\sim 0.5 \mathrm{~m})$ was typically two orders of magnitude larger than the Kolmogorov dissipative length scale $r_{d}$, and the extent of the observed inertial subrange reached the decade of scales during daytime. A strong correlation of $\varepsilon$ with the solar radiation rate is observed. The time delay of $\sim 1$ hour between the forcing by radiation and the dissipation roughly corresponds to the convective time macroscale.

\footnotetext{
Acknowledgements The present study is a part of the Russian Foundation for Basic Research RFBR, projects 16-05-00436_a and 18-05-60291. GK was supported by the German Research Foundation (DFG Project IceBound KI-853/11-1). DB was supported by the FEEL Foundation, Fondation pour l'Etude des Eaux du Léman.
} 


\section{References}

1. Bouffard D, Boegman L (2013) A diapycnal diffusivity model for stratified environmental flows. Dynamics of Atmospheres and Oceans 61-62:1434, DOI 10.1016/j.dynatmoce.2013.02.002, URL http://www.sciencedirect. com/science/article/pii/S0377026513000237

2. Bouffard D, Zdorovennov R, Zdorovennova G, Pasche N, Wüest A, Terzhevik A (2016) Ice-covered Lake Onega: effects of radiation on convection and internal waves. Hydrobiologia 780(1):22-36, DOI 10.1007/s10750-016-2915-3, URL https://link.springer.com/article/10.1007/s10750-016-2915-3

3. Chasnov J, Tse K (2001) Turbulent penetrative convection with an internal heat source. Fluid Dynamics Research 28:397-421, DOI 10.1016/ S0169-5983(00)00037-X

4. Deardorff JW (1970) Convective Velocity and Temperature Scales for the Unstable Planetary Boundary Layer and for Rayleigh Convection. Journal of the Atmospheric Sciences 27(8):1211-1213, DOI 10.1175/1520-0469(1970) 027<1211:CVATSF > 2.0.CO;2, URL https://journals.ametsoc.org/doi/ abs $/ 10.1175 / 1520-0469 \% 281970 \% 29027 \% 3$ C1211\%3ACVATSF $\% 3 E 2.0 . C 0 \% 3 B 2$

5. Deardorff JW (1970) Preliminary Results from Numerical Integrations of the Unstable Planetary Boundary Layer. Journal of the Atmospheric Sciences 27(8):1209-1211, DOI 10.1175/1520-0469(1970)027<1209: PRFNIO>2.0.CO;2, URL https://journals.ametsoc.org/doi/abs/10. 1175/1520-0469\%281970\%29027\%3C1209\%3APRFNIO\%3E2.0.CO\%3B2

6. Farmer DM (1975) Penetrative convection in the absence of mean shear. Quarterly Journal of the Royal Meteorological Society 101(430):869-891, 
DOI 10.1002/qj.49710143011, URL https://rmets.onlinelibrary.wiley. com/doi/abs/10.1002/qj.49710143011

7. Goluskin D, Poel EPvd (2016) Penetrative internally heated convection in two and three dimensions. Journal of Fluid Mechanics 791(R6), DOI 10.1017/jfm.2016.69, URL https://www. cambridge.org/core/journals/journal-of-fluid-mechanics/article/ penetrative-internally-heated-convection-in-two-and-three-dimensions/ 6F:OCI)CF 4FCDFCE:O (C696FFF:3F:104I)A4F

8. Hunt JCR (1984) Turbulence structure in thermal convection and shear-free boundary layers. Journal of Fluid Mechanics 138:161-184, DOI 10.1017/S0022112084000070, URL https://www. cambridge.org/core/journals/journal-of-fluid-mechanics/article/ turbulence-structure-in-thermal-convection-and-shearfree-boundary-layers/ 17686F1476A274C2DO9DCED9FE95B139

9. Jonas T, Terzhevik AY, Mironov DV, Wüest A (2003) Radiatively driven convection in an ice-covered lake investigated by using temperature microstructure technique. Journal of Geophysical Research: Oceans 108(C6), DOI 10.1029/2002JC001316, URL https://agupubs.onlinelibrary.wiley.com/ doi/abs/10.1029/2002JC001316

10. Kadanoff LP (2001) Turbulent Heat Flow: Structures and Scaling. Physics Today 54(8):34-39, DOI 10.1063/1.1404847, URL https://physicstoday. scitation.org/doi/10.1063/1.1404847

11. Kaimal JC, Wyngaard JC, Haugen DA, Coté OR, Izumi Y, Caughey SJ, Readings CJ (1976) Turbulence Structure in the Convective Boundary Layer. Journal of the Atmospheric Sciences 33(11):2152- 
2169, DOI $10.1175 / 1520-0469(1976) 033<2152:$ TSITCB $>2.0$. CO $; 2, \quad$ URL https://journals.ametsoc.org/doi/abs/10.1175/1520-0469\%281976\% $29033 \% 3 \mathrm{C} 2152 \% 3 \mathrm{ATSITCB} \% 3 \mathrm{E} 2.0 . \mathrm{CO} \% 3 \mathrm{~B} 2$

12. Kirillin G, Aslamov I, Leppäranta M, Lindgren E (2018) Turbulent mixing and heat fluxes under lake ice: the role of seiche oscillations. Hydrology and Earth System Sciences Discussions pp 1-22, DOI https://doi.org/10. 5194/hess-2018-376, URL https://www.hydrol-earth-syst-sci-discuss. net/hess-2018-376/

13. Kirillin GB, Forrest AL, Graves KE, Fischer A, Engelhardt C, Laval BE (2015) Axisymmetric circulation driven by marginal heating in icecovered lakes. Geophysical Research Letters 42(8):2893-2900, DOI 10.1002/ 2014GL062180, URL https://agupubs.onlinelibrary.wiley.com/doi/abs/ $10.1002 / 2014$ GL062180

14. Kolmogorov AN (1941) The Local Structure of Turbulence in Incompressible Viscous Fluid for Very Large Reynolds Numbers. Proceedings: Mathematical and Physical Sciences 434(1890), URL http://www.jstor.org/stable/51980

15. Lenschow DH, Stephens PL (1980) The role of thermals in the convective boundary layer. Boundary-Layer Meteorology 19(4):509-532, DOI 10.1007/BF00122351, URL https://link.springer.com/article/10.1007/ BF00122351

16. Lhermitte R, Serafin R (1984) Pulse-to-Pulse Coherent Doppler Sonar Signal Processing Techniques. Journal of Atmospheric and Oceanic Technology 1(4):293-308, DOI 10.1175/1520-0426(1984)001<0293:PTPCDS > 2.0. CO;2, URL https://journals.ametsoc.org/doi/abs/10.1175/1520-0426\% $281984 \% 29001 \% 3 C 0293 \% 3$ APTPCDS $\% 3 E 2.0 . C 0 \% 3 B 2$ 
17. Lilly DK (1983) Stratified Turbulence and the Mesoscale Variability of the Atmosphere. Journal of the Atmospheric Sciences 40(3):749761, DOI $10.1175 / 1520-0469(1983) 040<0749:$ STATMV $>2.0 . C O ; 2, \quad$ URL https://journals.ametsoc.org/doi/abs/10.1175/1520-0469\%281983\% 29040\%3C0749\%3ASTATMV\%3E2.0.CO $\% 3 B 2$

18. Lohse D, Xia KQ (2010) Small-Scale Properties of Turbulent RayleighBénard Convection. Annual Review of Fluid Mechanics 42(1):335-364, DOI 10. 1146/annurev.fluid.010908.165152, URL https://www.annualreviews.org/ doi/10.1146/annurev.fluid.010908.165152

19. Lombardo CP, Gregg MC (1989) Similarity scaling of viscous and thermal dissipation in a convecting surface boundary layer. Journal of Geophysical Research: Oceans 94(C5):6273-6284, DOI 10.1029/ JC094iC05p06273, URL https://agupubs.onlinelibrary.wiley.com/doi/ abs/10.1029/JC094iC05p06273

20. Lorke A, Wüest A (2005) Application of Coherent ADCP for Turbulence Measurements in the Bottom Boundary Layer. Journal of Atmospheric and Oceanic Technology 22(11):1821-1828, DOI 10.1175/JTECH1813.1, URL https://journals.ametsoc.org/doi/abs/10.1175/JTECH1813.1

21. Mironov D, Terzhevik A, Kirillin G, Jonas T, Malm J, Farmer D (2002) Radiatively driven convection in ice-covered lakes: Observations, scaling, and a mixed layer model. Journal of Geophysical Research: Oceans 107(C4):7-17-16, DOI 10.1029/2001JC000892, URL https://agupubs.onlinelibrary. wiley.com/doi/abs/10.1029/2001JC000892

22. Monin AS, Yaglom AM (2013) Statistical Fluid Mechanics, Volume II: Mechanics of Turbulence. Courier Corporation 
23. Nieuwstadt FTM, Brost RA (1986) The Decay of Convective Turbulence. Journal of the Atmospheric Sciences 43(6):532-546, DOI 10.1175/1520-0469(1986) $043<0532$ :TDOCT > 2.0.CO;2, URL https://journals.ametsoc.org/doi/ abs $/ 10.1175 / 1520-0469(1986) 043 \% 3$ C0532\%3ATDOCT\%3E2.0.CO\%3B2

24. Obukhov AM, Yaglom AM (1959) On the microstructure of atmospheric turbulence A review of recent work in the U.S.S.R. Quarterly Journal of the Royal Meteorological Society 85(364):81-90, DOI 10.1002/qj. 49708536402, URL https://rmets.onlinelibrary.wiley.com/doi/abs/10. $1002 / q j \cdot 49708536402$

25. Pandey A, Scheel JD, Schumacher J (2018) Turbulent superstructures in Rayleigh-Bénard convection. Nature Communications 9(1):2118, DOI 10.1038/s41467-018-04478-0, URL https://www.nature.com/articles/ $54146 /-018-044 / 8-0$

26. Shay TJ, Gregg MC (1986) Convectively Driven Turbulent Mixing in the Upper Ocean. Journal of Physical Oceanography 16(11):17771798, DOI 10.1175/1520-0485(1986)016<1777:CDTMIT >2.0.CO;2, URL https://journals.ametsoc.org/doi/abs/10.1175/1520-0485\%281986\% $29016 \% 3 \mathrm{C} 1777 \% 3 \mathrm{ACDTMIT} \% 3 \mathrm{E} 2.0 . \mathrm{CO} \% 3 \mathrm{~B} 2$

27. Sreenivasan KR (1995) On the universality of the Kolmogorov constant. Physics of Fluids 7(11):2778-2784, DOI 10.1063/1.868656, URL https://aip. scitation.org/doi/10.1063/1.868656

28. Straus M (1976) Penetrative convection in a layer of fluid heated from within. The Astrophysical Journal 209:179-189, DOI 10.1086/154707

29. Turner JS (1973) Buoyancy Effects in Fluids by J. S. Turner. DOI 10.1017/ CBO9780511608827, URL /core/books/buoyancy-effects-in-fluids/ 
30. Ulloa HN, Wüest A, Bouffard D (2018) Mechanical energy budget and mixing efficiency for a radiatively heated ice-covered waterbody. Journal of Fluid Mechanics 852:R1, DOI 10.1017/jfm.2018.587

31. Wiles PJ, Rippeth TP, Simpson JH, Hendricks PJ (2006) A novel technique for measuring the rate of turbulent dissipation in the marine environment. Geophysical Research Letters 33(L21608), DOI 10.1029/ 2006GL027050, URL https://agupubs .onlinelibrary.wiley.com/doi/abs/ $10.1029 / 2006$ GL027050

32. Zdorovennov R, Palshin N, Zdorovennova G, Efremova T, Terzhevik A (2013) Interannual variability of ice and snow cover of a small shallow lake. Estonian Journal of Earth Sciences 62(1):26-32, DOI 10.3176/earth.2013.03

33. Zdorovennov R, Gavrilenko G, Zdorovennova G, Palshin N, Efremova T, Golosov S, Terzhevik A (2016) Optical properties of lake Vendyurskoe. Geography, Environment, Sustainability 9(3):74-87, DOI 10.15356/2071-9388

542 03v09_2016_05

34. Zilitinkevich S (1991) Turbulent Penetrative Convection. Avebury Technical, Aldershot 